\title{
High-efficiency Separation of Extracellular Vesicles from Lipoproteins in Plasma by Agarose Gel Electrophoresis
}

\author{
Yan Zhang ${ }^{1,4,5^{\square}}$, Zaian Deng ${ }^{1,6}{ }^{\square}$, Doudou Lou $^{1 \square}$, Yong Wang ${ }^{3}$, Rui Wang ${ }^{4}$, Rui Hü ${ }^{4}$ Xueer Zhang ${ }^{4}$, Qingfu \\ $\mathrm{Zhu}^{1 *}$, Yuchao Chen ${ }^{1,2,3 *}$, Fei Liu ${ }^{1,3 *}$ \\ 1. School of Ophthalmology \& Optometry, School of Biomedical Engineering, Wenzhou Medical University, Wenzhou, Zhejiang \\ 325035, China \\ 2. The First Affiliated Hospital of Wenzhou Medical University, Wenzhou, Zhejiang 325035, China \\ 3. Wenzhou Institute, University of Chinese Academy of Sciences, Wenzhou, Zhejiang 325000, China \\ 4. Department of Preventive Medicine, Wenzhou Medical University, Wenzhou, Zhejiang 325035, China \\ 5. Department of Preventive Medicine, School of Medicine, Hangzhou Normal University, Hangzhou 310018, China \\ 6. College of Health Science and Environmental Engineering, Shenzhen Technology University, Shenzhen, Guangdong 518118, \\ China \\ *E-mail: Qingfu Zhu, qingfu.zhu@wmu.edu.cn; Yuchao Chen, yuchaochen@wellsimbiotech.com; Fei Liu, \\ liufei2017@wmu.edu.cn
}

\section{Table of Contents}

1. The performance of PKH67 and PKH67 labeled EVs in agarose gel electrophoresis....................

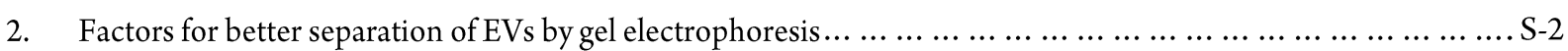

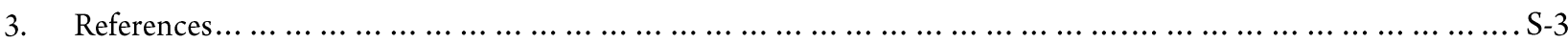




\section{The performance of PKH67 and PKH67 labeled EVs in agarose gel electrophoresis}

EVs and lipoproteins were both labeled with PKH67 fluorescent dye. As shown in Figure S1a, when the PKH67 was free, it could not migrate in agarose gel and remained in the sample loading well. The labeling of PKH67 did not cause the destruction of EVs. As a contrast, 1\% sodium dodecyl sulphate (SDS) was used to treat EV samples, which destroyed EVs by denaturing proteins and resulted in a significantly increase in migration velocity.

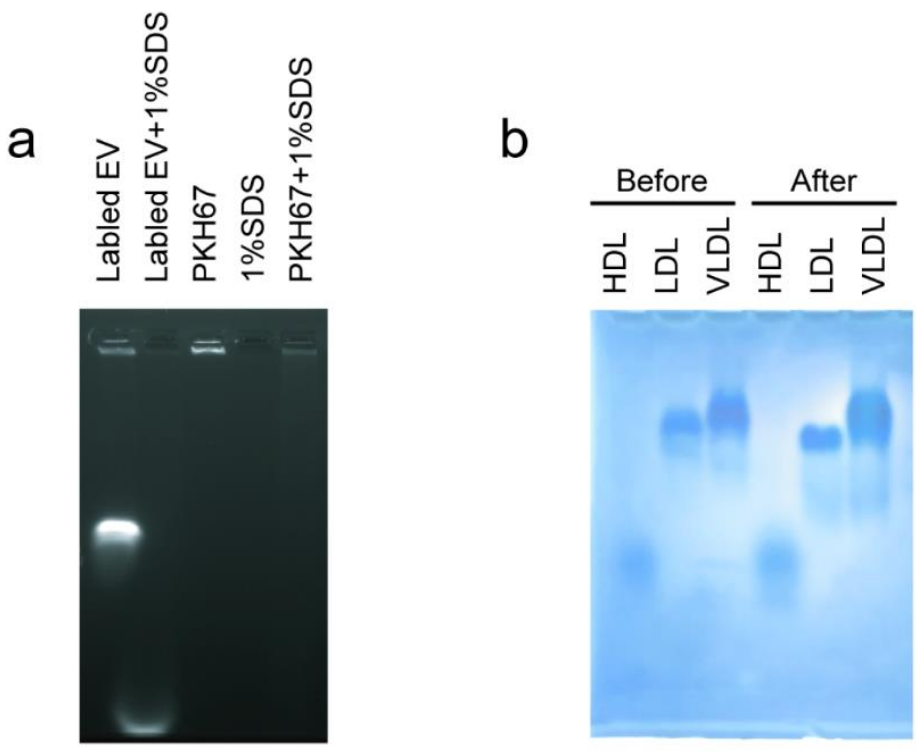

Figure S1. Photograph of agarose gel electrophoresis (TAE buffer $\mathrm{pH}=7.4$, agarose gel concentration $1 \%, 100 \mathrm{~V}, 2 \mathrm{~h}$ ). Loading wells were located below the edge of the top. (a) PKH67 labeled EVs. Images were captured by a fluorescent chemiluminescence gel imaging system. (b) Sudan Black B labeled lipoproteins before or after electrophoresis. Images were captured using an iphone 6 S's built-in camera.

\section{Factors for better separation of EVs by gel electrophoresis}

According to the electrokinetic theory, the efficiency of electrophoresis depends on three factors: electrophoretic mobility $\left(\mu_{\mathrm{ep}}\right)$, Electroosmotic flow of the bulk solution (EOF), and joule heating. Electrophoretic mobility $\left(\mu_{\mathrm{ep}}\right)$ mainly depends on the charge-to-size-ratio of the sample. The direction of electrophoresis in agarose gel not only depends on the electrophoresis, but also the electroosmosis. Apart from the $\mathrm{pH}$ of buffer and the concentration of agarose gel discussed in the manuscript, some other conditions have also been optimized to get better results of EV separation.

First, the amount of plasma loaded into the wells should not be too high. Too much plasma will result in distorted bands of lipoproteins and EVs after electrophoresis.

The power supply of the electrophoresis system is also vital for the experiment. As it is reported by Michael $\mathrm{Tu}$ et al, a cyclic square wave electric field could induce a release of exosome biomarker inside EVs ${ }^{1}$. If the voltage is too high, there is a significant possibility that the EVs will degrade and/or release their contents. However, if the voltage is too low, it would not only increase the electrophoresis time, but also cause the diffusion of the sample and finally lead to the low separation efficiency. In our experiment, the electrophoresis voltage was set at $100 \mathrm{~V}$. In additiona, a considerable amount of heat can be produced during electrophoresis (especially in EV recovery procedure) and overheating will result in the denaturation of EVs. To minimize the side effects of joule heating, our electrophoresis system was bathed in ice.

The choice of running buffer and agarose greatly affect the migration of EVs. For example, in another traditional running buffer, phenobarbital buffer, EVs cannot be separated from lipoproteins effectively. 
Furthermore, the agarose used in this study exhibited low electrodosmosis, since unexpected electrodosmosis in agarose can inevitably cause side effects on electrophoresis.

\section{References}

1 Wei, F.; Yang, J.; Wong, D. T. Detection of exosomal biomarker by electric field-induced release and measurement (EFIRM). Bionsens. Bioelectron. 2013, 44, 115-121. 